\title{
Plasmonics in the UV range with Rhodium nanocubes
}

\author{
X. Zhang ${ }^{\mathrm{a}}$, Y. Gutiérrez ${ }^{\mathrm{b}}$, P. Li ${ }^{\mathrm{a}}$, Á. I. Barreda ${ }^{\mathrm{b}}$, A. M. Watson ${ }^{\mathrm{a}}$, R. Alcaraz de la Osa ${ }^{\mathrm{b}}$, G. \\ Finkelstein ${ }^{\mathrm{c}}$, F. González ${ }^{\mathrm{b}}$, D. Ortiz ${ }^{\mathrm{b}}$, J. M. Saiz ${ }^{\mathrm{b}}$, J. M. Sanz ${ }^{\mathrm{b}}$, H. O. Everitt ${ }^{\mathrm{c}, \mathrm{d}}$, J. Liu ${ }^{\mathrm{a}}$, and \\ F. Moreno ${ }^{\mathrm{b}}$

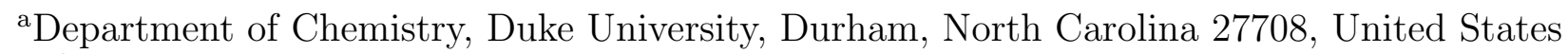 \\ ${ }^{b}$ Departamento de Física Aplicada, Universidad de Cantabria 39005, Santander, Spain \\ ${ }^{c}$ Department of Physics, Duke University, Durham, North Carolina 27708, United States \\ dArmy Aviation and Missile RD\&E Center, Redstone Arsenal, Alabama 35898, United States
}

\begin{abstract}
Plasmonics in the UV-range constitutes a new challenge due to the increasing demand to detect, identify and destroy biological toxins, enhance biological imaging, and characterize semiconductor devices at the nanometer scale. Silver and aluminum have an efficient plasmonic performance in the near UV region, but oxidation reduces its performance in this range. Recent studies point out rhodium as one of the most promising metals for this purpose: it has a good plasmonic response in the UV and, as gold in the visible, it presents a low tendency to oxidation. Moreover, its easy fabrication through chemical means and its potential for photocatalytic applications, makes this material very attractive for building plasmonic tools in the UV. In this work, we will show an overview of our recent collaborative research with rhodium nanocubes (NC) for Plasmonics in the UV.
\end{abstract}

Keywords: UV plasmonics, LPSR, Rhodium, nanocube.

\section{INTRODUCTION}

Plasmonics is an active branch of Nanophotonics which studies the distribution of the electromagnetic field, and its local charge resonances (Localized Surface Plasmon Resonances, LSPRs) in subwavelength metallic nanostructures when electromagnetically irradiated. ${ }^{1}$ In particular, nanoparticles made of metals like gold and silver have given rise to plasmonic tools with many interesting and useful applications at infrared and optical frequencies: ${ }^{2}$ biomedicine, communications, spectroscopy techniques, etc.

At present, the UV-range constitutes a new focus of attention for Plasmonics because of the many challenges arising in fields like biology or semiconductor technology. ${ }^{3-5}$ Silver has an efficient plasmonic response in the near UV region, but oxidation reduces its performance in this range. A study by J. M. Sanz et al. ${ }^{6}$ analyzed several metals in order to find those most promising for UV plasmonics. From this work, those who showed more compelling properties for this purpose were aluminum $(\mathrm{Al})$, gallium $(\mathrm{Ga})$, magnesium $(\mathrm{Mg})$ and rhodium $(\mathrm{Rh})$. Aluminum ${ }^{7,8}$ has proven to be a good candidate because its bulk plasma frequency is around $13 \mathrm{eV}$. In addition, it has high natural abundance, low cost and can be processed by many methods. However, this metal oxidizes even more rapidly than silver, so it is difficult to implement effective and stable nano-devices if they are exposed to aqueous environments. On the one hand, as the proportion of oxide increases, a decrease of the scattering efficiency and a red-shift of the resonances are produced. ${ }^{9}$ On the other hand, the reduction of the percetage of metal, leads to a blue-shift of the LSPR. So, the presence of an oxidation layer has a critical importance when reproducible plasmonic behaviour is required. Magnesium ${ }^{10}$ also has very promising properties for plasmonics applications. It has been presented as an alternative to aluminum because it provides higher extinction efficiencies in the same wavelength range. However, $\mathrm{Mg}$ also suffer from the formation of an oxide layer that diminishes its plasmonic activity. As a promising alternative, nanoparticles made of gallium ${ }^{11-14}$ have been recently proposed for SERS (Surface Enhanced Raman Scattering) experiments. Its main advantage is that, when they are exposed to the atmosphere, Ga nanoparticles form a thin, self-terminating oxide layer that

Further author information: (Send correspondence to F.M)

F.M.: E-mail: morenof@unican.es

Nanophotonics VI, edited by David L. Andrews, Jean-Michel Nunzi, Andreas Ostendorf, Proc. of SPIE Vol. 9884, 98841E · C 2016 SPIE · CCC code: 0277-786X/16/\$18 · doi: 10.1117/12.2227674 
protects the pure core. As a result, this thin layer provides structural and chemical stability that allows them to keep stable optical response over months. However, this metal presents a solid-liquid phase transition at room temperature that hinders its manipulation for other kind of applications. One of the main conclusion ${ }^{6}$ is that rhodium ${ }^{15}$ is a very promising metal, not only for its plasmonic behavior in the near UV but also, as gold in the visible, it is a noble metal with low tendency to oxidation. Moreover, its easy fabrication through chemical means (for instance with tripod ${ }^{15,16}$ and cube $^{17}$ shapes and sizes smaller than $10 \mathrm{~nm}$ ) and its potential for photo-catalytic applications, makes this material very attractive for building plasmonic tools in the UV.

In the present contribution, the electromagnetic behaviour of isolated Rh nanocubes (NCs) of different side lengths (from 10 to $60 \mathrm{~nm}$ ) has been numerically studied and compared with experimental measurements made by UV-VIS extinction spectroscopy. The effect of introducing some deviations from the perfect shape has been also analyzed, as well as cooperative and coupling effects between neighbouring NCs.

This work is organized as follows: in Section 2 we briefly review the theoretical methods and the geometry of the studied systems is described. In Section 3, we show the main results of this research. Finally, in Section 4, the main conclusions are presented.

\section{THEORETICAL METHODS AND SYSTEM GEOMETRY}

The electromagnetic interaction of light with Rh nanoparticles (NPs) has been modelled by means of finiteelements method (FEM) simulations. For the implementation, we have chosen COMSOL Multiphyisics 4.4. In particular, we used the RF Module that allows us to formulate and solve the differential form of Maxwell equations with boundary conditions.

The absorption cross-section $C_{a b s}$ can be calculated as the integral of the resisitive losses over the NP's volume, normalized to the incident power density. The scattering cross-section $C_{s c a}$ is derived by integrating the Poynting vector over an imaginary sphere, whose radius is bigger than the illuminanting wavelength, around the NP. Once again, the normalization is done to the incident power density. The absorption and scattering efficiencies, $Q_{a b s}$ and $Q_{s c a}$, are defined by

$$
Q_{a b s}=\frac{C_{a b s}}{S} \quad Q_{s c a}=\frac{C_{s c a}}{S}
$$

where $S$ is the NP cross-sectional area projected onto a plane perpendicular to the propagation of the incident beam (e.g. $S=l^{2}$ for a cube of edge length $l$ ). The spectral extinction efficiency $Q_{e x t}$ was calculated as the sum of the absorption and scattering efficiencies.

Experimentally, the optical properties of Rh NCs have been explored through UV-vis extinction spectroscopy.

We have performed finite-element simulation over different Rh NCs structures: isolated cubes and dimers. All of them have been illuminated with a monochromatic plane wave with wave vector $\vec{k}$ along the Z-axis and have been considered to be embedded in ethanol $\left(\epsilon_{\text {ethanol }}=1.96\right.$, the medium used in the experiment). In the case of the isolated cube, it has been illuminated by one of its faces with a polarization along X-axis (Fig. 1.a). The dimer has been iluminated with both X- and Y-polarizations (Fig. 1.b).

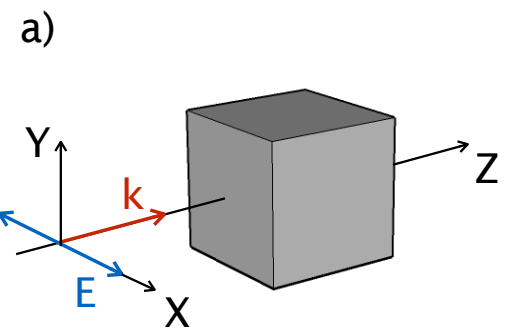

b)

Figure 1. Scattering geometries: (a) Isolated Rh NC and (b) Rh NCs dimer. 
The dielectric function of Rhodium has been obtained from the literature. ${ }^{18}$ Figure 2 show the real and imaginary parts of the relative electric permittivity of Rh.

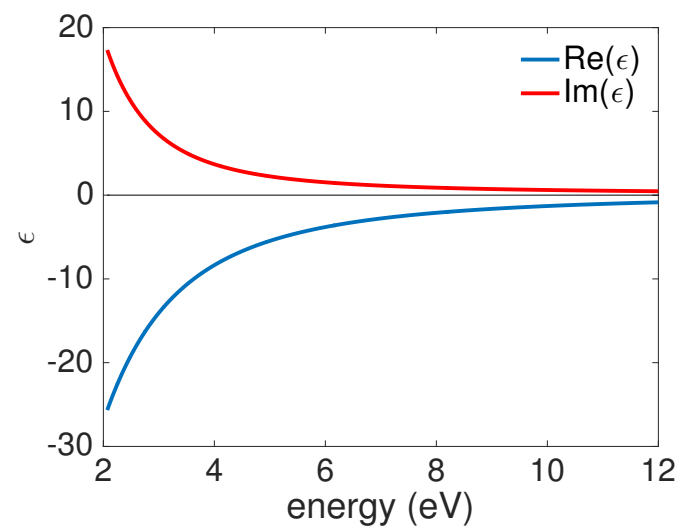

Figure 2. Real (blue line) and imaginary (red line) parts of the relative electric permittivity $\epsilon$ for Rh as a function of the incident energy.

\section{RESULTS}

In this section, the UV spectral response of isolated Rh NCs are studied and compared with experimental measurements. Moreover, the effect of some deviations from the perfect shape are also analyzed. In order to be able to study cooperative and coupling effects between the nanocubes, the simplest aggregate system, the dimer, is also analyzed.

\subsection{Isolated $\mathrm{Rh}$ nanocubes}

As a first step we consider isolated cubes of different size embedded in ethanol. Fig 3.a shows the extinction effiencies of Rh NCs, with edge lengths ranging from 3 to $59 \mathrm{~nm}$, as a function of the incident energy. As the size of the NC increases, $Q_{e x t}$ takes larger values and the LSPR is shifted towards smaller energies. Fig. 3.b shows the agreement between the experimental (blue dots) and numerical solutions (red dots) of the LSPR energies of Rh NCs in ethanol.
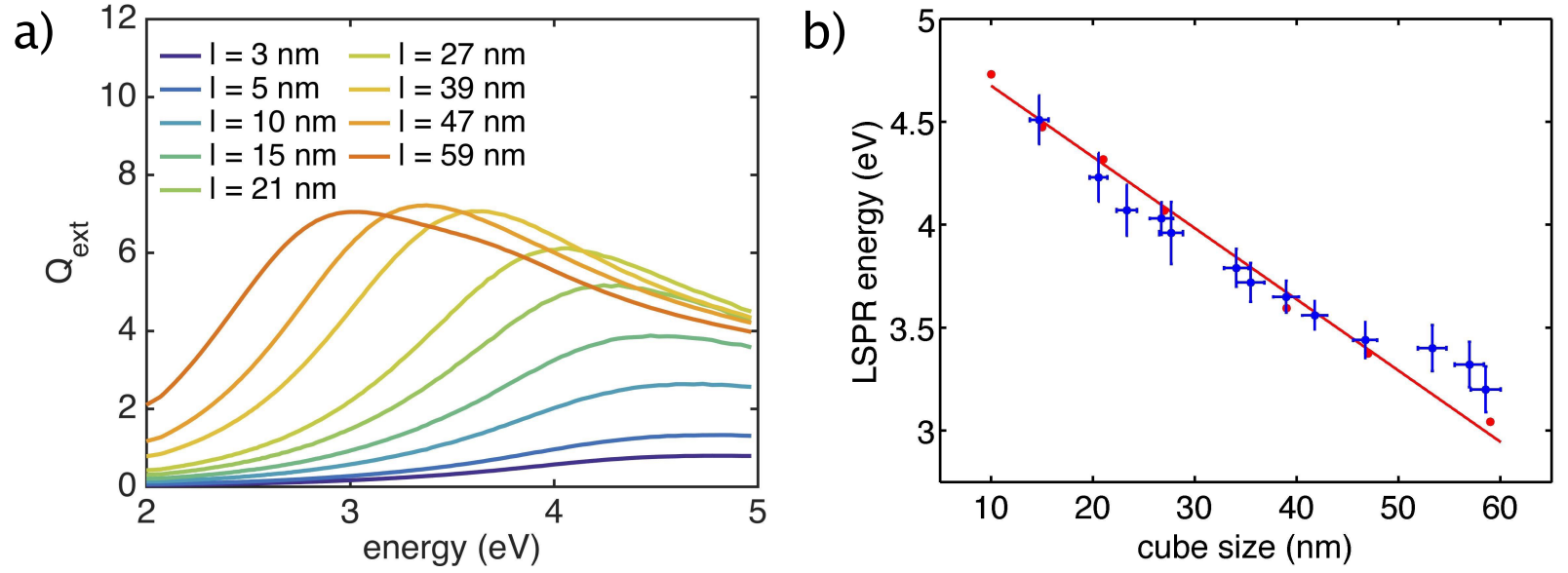

Figure 3. (a) Extinction effiencies of Rh NCs of different edge lengths $l$ ranging from 3 to 59 nm. (b) Relationship of the cube sizes and LSPR energies from experimental measurements (blue dots) and numerical simulations (red dots) of Rh NCs in ethanol. The red solid line is a linear fitting of the simulated results. 
Figure 4 shows the near field intensity distribution, in terms of the square modulus of the electric field $|E|^{2}$ at the surface of a $39 \mathrm{~nm} \mathrm{Rh} \mathrm{NC}$ at the resonant energy. As it can be seen, the value $|E|^{2}$ is larger at the vertices of the cube, being almost zero at the center of the faces.

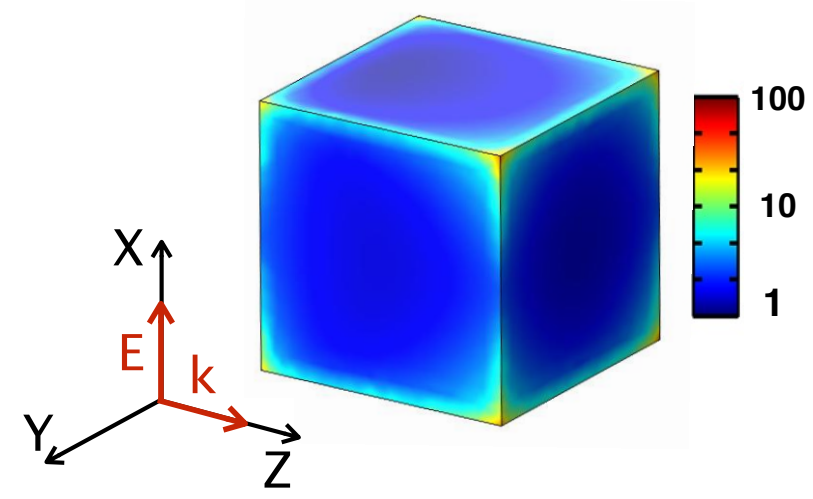

Figure 4. Distribution of $|E|^{2}$ at the surface of a $39 \mathrm{~nm} \mathrm{Rh} \mathrm{NC}$ at the resonant energy $3.6 \mathrm{eV}$, along with the coordinates used for the finite element modeling. The light is polarized along the $\mathrm{X}$-axis and propagating in $\mathrm{Z}$-axis in the simulation.

This study has been inspired by the work on the chemical synthesis of Rh NCs. ${ }^{17}$ Experimentally, it is almost impossible to obtain perfect cubes. For that reason, the effect on the $Q_{a b s}$, a mesureable magnitude, of stretching the cube parallel (X-direction) and perpendicular (Y-and Z-directions) to the polarization of the illuminating beam has been studied (see Fig. 5).

When the stretching is parallel to the polarization direction (red line), a red-shift of the LSPR is produced. This effect is a natural consequence of the increasing cross-section. However, when the stretching is perpedicular to the polarization direction (blue and green lines), a blue-shift of the LSPR is produced. These blue-shifts can be interpreted by means of the harmonic oscillator model for localized plasmonic excitations, where the resonance frequency is proportional to the restoring force experienced by the displaced electrons on one side of the NP caused by the background of exposed positive ions on the other side. ${ }^{19}$ When the NPs edges that are perpendicular to the displacement become longer, the amount of charge driven by the incident electric field increases. However, since their separation remains unchanged, the displaced electron gas experiences a larger restoring force, and the resonance blue-shifts to higher energies.

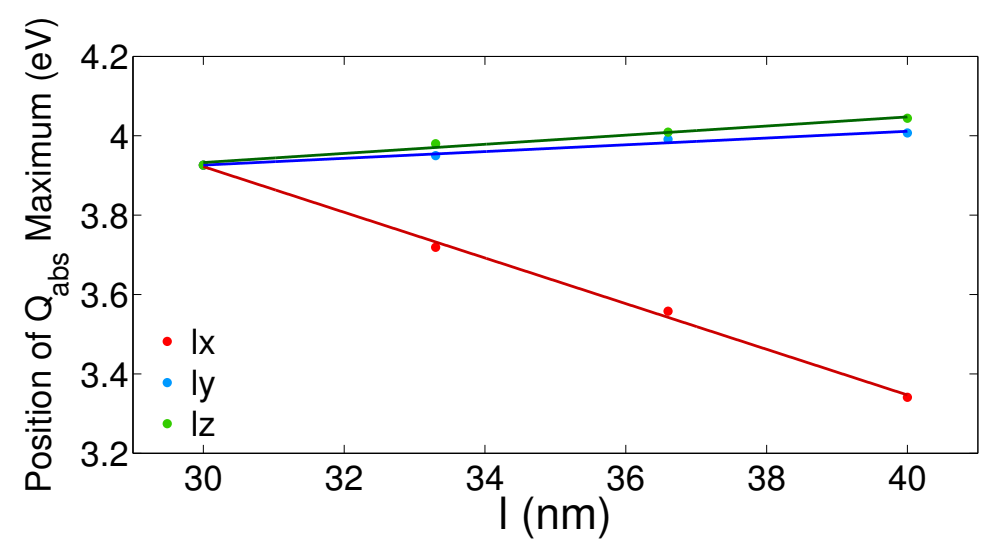

Figure 5. Comparative of the evolution of the energy at which the electric dipolar resonance is produced when a $l=30$ $\mathrm{nm}$ side Rh NC is streched along the X- (red dots), Y- (blue dots) and Z-axis (green dots). The solid lines are the linear fittings of the simulated results. 


\subsection{Rh nanocubes dimer}

Figure 6 shows the extinction efficiency $Q_{\text {ext }}$ of a cubic symmetric dimer. The cubes have been considered to have a side length $l$ of $30 \mathrm{~nm}$. Different gaps have been studied ranging from 5 to $130 \mathrm{~nm}$. Both X- and Ypolarization have been considered (see Fig. 1.b).
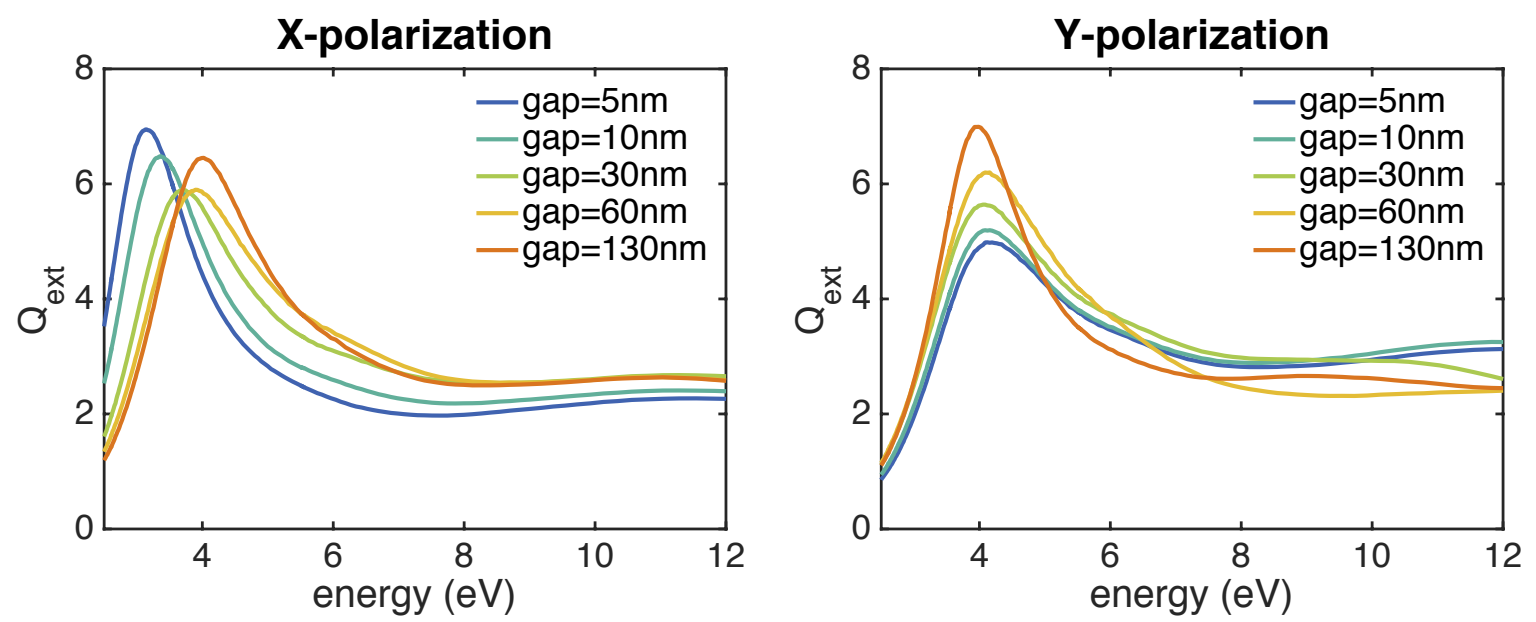

Figure 6. Spectral extinction efficiency $Q_{\text {ext }}$ for Rh cubic dimers of several gaps, varying from 5 to $130 \mathrm{~nm}$, illuminated under normal X- and Y-polarizations.

Depending on the polarization direction, the LSPR is either shifted to higher (Y-polarization) or lower energies (X-polarization) when the gap becomes smaller. These effects were explained by Rechberger et al. ${ }^{20}$ When the illuminating beam is polarized perpendicular to the dimer's axis (Y-polarization), the charge distributions inside each NP act cooperatively to enhance the repulsive action inside both particles. As a result, a blue-shift of the resonant frequency is produced. However, when the polarization is parallel to the dimer's axis (X-polarization), a weakening of the repulsive forces inside the spheres is experienced and a red-shift of the LSPR occurs.

\section{CONCLUSIONS}

Due to the possibility of manufacturing rhodioum nanocubes, the UV plasmonic properties of various Rh NCs geometries have been theoretically explored. The theoretical results obtained for isolated $\mathrm{Rh} \mathrm{NCs}$ have been compared with experimental measurements done by UV-vis extinction spectroscopy. Because the synthesis of totally perfect cubes is almost experimentally impossible, some deviations from the perfect shape has been analyzed. Whereas stretching the cube in the polarization direction produces a red-shift of the LSPRs, the stretching in the perpendicular directions leads to a blue-shift of the LSPRs. Finally, the simplest system in which interaction and coupling effects are produced, the dimer, has been studied. Polarizing the illuminating beam paralel/perpendicular to the particle axis produces a red-/blue-shift of the LSPRs as the gap diminishes.

\section{ACKNOWLEDGMENTS}

This research was partly supported by MICINN (Spanish Ministry of Science and Innovation) through project FIS2013-45854-P. Y.G and Á.I.B want to thank the University of Cantabria for their FPU grants.

\section{REFERENCES}

[1] Prasad, P. N., [Nanophotonics], John Wiley \& Sons, Inc. (2004).

[2] Giannini, V., Fernández-Domínguez, A. I., Heck, S. C., and Maier, S. a., "Plasmonic nanoantennas: Fundamentals and their use in controlling the radiative properties of nanoemitters," (2011). 
[3] Anker, J. N., Hall, W. P., Lyandres, O., Shah, N. C., Zhao, J., and Van Duyne, R. P., "Biosensing with plasmonic nanosensors.," Nature materials 7(6), 442-453 (2008).

[4] Chowdhury, M. H., Ray, K., Gray, S. K., Pond, J., and Lakowicz, J. R., "Aluminum nanoparticles as substrates for metal-enhanced fluorescence in the ultraviolet for the label-free detection of biomolecules," Anal. Chem. 81(4), 1397-1403 (2009).

[5] Taguchi, A., Hayazawa, N., Furusawa, K., Ishitobi, H., and Kawata, S., "Deep-UV tip-enhanced Raman scattering," Journal of Raman Spectroscopy 40(9), 1324-1330 (2009).

[6] Sanz, J. M., Ortiz, D., Alcaraz de la Osa, R., Saiz, J. M., González, F., Brown, a. S., Losurdo, M., Everitt, H. O., and Moreno, F., "UV Plasmonic Behavior of Various Metal Nanoparticles in the Near- and Far-Field Regimes: Geometry and Substrate Effects," The Journal of Physical Chemistry C 117(38), 19606-19615 (2013).

[7] Knight, M. W., Liu, L., Wang, Y., Brown, L., Mukherjee, S., King, N. S., Everitt, H. O., Nordlander, P., and Halas, N. J., "Aluminum plasmonic nanoantennas.," Nano letters 12(11), 6000-6004 (2012).

[8] Knight, M. W., King, N. S., Liu, L., Everitt, H. O., Nordlander, P., and Halas, N. J., "Aluminum for plasmonics," ACS Nano 8(1), 834-840 (2014).

[9] Chan, G. H., Zhao, J., Schatz, G. C., and Duyne, R. P. V., "Localized surface plasmon resonance spectroscopy of triangular aluminum nanoparticles," Journal of Physical Chemistry C 112(36), 13958-13963 (2008).

[10] Sterl, F., Strohfeldt, N., Walter, R., Griessen, R., Tittl, A., and Giessen, H., "Magnesium as Novel Material for Active Plasmonics in the Visible Wavelength Range," Nano Letters, 150827121228003 (2015).

[11] Albella, P., Garcia-Cueto, B., González, F., Moreno, F., Wu, P. C., Kim, T. H., Brown, A., Yang, Y., Everitt, H. O., and Videen, G., "Shape matters: Plasmonic nanoparticle shape enhances interaction with dielectric substrate," Nano Letters 11(9), 3531-3537 (2011).

[12] Wu, P. C., Losurdo, M., Kim, T. H., Garcia-Cueto, B., Moreno, F., Bruno, G., and Brown, A. S., "GaMg Core-shell nanosystem for a novel full color plasmonics," Journal of Physical Chemistry C 115(28), 13571-13576 (2011).

[13] Yang, Y., Akozbek, N., Sanz, J. M., Losurdo, M., Brown, A. S., Everitt, H. O., Kim, T.-h., and Moreno, F., "Ultraviolet-visible plasmonic properties of gallium nanoparticles investigated by variable angle spectroscopic and Mueller matrix ellipsometry variable angle spectroscopic and Mueller matrix ellipsometry," (2014).

[14] Knight, M. W., Coenen, T., Yang, Y., Brenny, B. J. M., Losurdo, M., Brown, A. S., Everitt, H. O., and Polman, A., "Gallium Plasmonics: Deep Subwavelength Spectroscopic Imaging of Single and Interacting Gallium Nanoparticles," ACS Nano 9(2), 2049-2060 (2015).

[15] Watson, A. M., Zhang, X., Alcaraz de la Osa, R., Marcos Sanz, J., González, F., Moreno, F., Finkelstein, G., Liu, J., and Everitt, H. O., "Rhodium nanoparticles for ultraviolet plasmonics.," Nano letters 15(2), 1095-100 (2015).

[16] Alcaraz de la Osa, R., Sanz, J. M., Barreda, a. I., Saiz, J. M., González, F., Everitt, H. O., and Moreno, F., "Rhodium Tripod Stars for UV Plasmonics," The Journal of Physical Chemistry C 119(22), 12572-12580 (2015).

[17] Zhang, X., Li, P., Barreda, Á., Gutiérrez, Y., González, F., Moreno, F., Everitt, H., and Liu, J., "Size-Tunable Rhodium Nanostructures for Wavelength-Tunable Ultraviolet Plasmonics," Nanoscale Horiz. (2016).

[18] Palik, E., [Handbook of Optical Constants of Solids], Academic Press (1998).

[19] Henson, J., Dimaria, J., and Paiella, R., "Influence of nanoparticle height on plasmonic resonance wavelength and electromagnetic field enhancement in two-dimensional arrays," Journal of Applied Physics 106(9), 093111 (2009).

[20] Rechberger, W., Hohenau, A., Leitner, A., Krenn, J. R., Lamprecht, B., and Aussenegg, F. R., "Optical properties of two interacting gold nanoparticles," Optics Communications 220(1-3), 137-141 (2003). 